\title{
Cuadernos de Historia de la Salud Pública en su 55 Aniversario
}

\author{
55th anniversary of Cuadernos Historia de la Salud Pública
}

\section{Gregorio Delgado García}

Historiador Médico del MINSAP. Escuela Nacional de Salud Pública. La Habana, Cuba.

\section{NTRODUCCI ÓN}

Al fundar el académico César Rodríguez Expósito (1904-1972) la Oficina del Historiador del Ministerio de Salud Pública en 1951, creyó oportuno que tuviera un órgano publicitario en el que se recogiera su labor de investigación y de recopilación de información sobre el desarrollo histórico de la salud pública en el país.

De ahí surgió la colección monográfica Cuadernos de Historia Sanitaria en 1952, que nueve años más tarde cambiaba ligeramente su nombre por el de Cuadernos de Historia de la Salud Pública, con el que se mantiene en la actualidad.

Al surgir la colección, era la tercera que existía en el país, la primera Cuadernos de Historia Habanera fundada en 1935 por el doctor Emilio Roig de Leuchsenring (1889-1964) no logró sobrevivir al fallecimiento de su iniciador y único director y la segunda, Cuadernos de Cultura, debida al entusiasmo creador del doctor José María Chacón y Calvo (1892-1969) en su etapa de Director de Cultura de la Secretaría de Educación, después Ministerio de Educación, quien la fundó en 1938. El doctor Raúl Roa García (1907-1982) que lo sustituyó en el cargo la mantuvo, pero el doctor Guillermo de Zendegui Carbonell (1909- ?), que ocupó dicha plaza durante la dictadura batistiana la dio por terminada.

Con el triunfo revolucionario Cuadernos de Historia de la Salud Pública* no sólo se mantuvo como única colección en el país, sino que aumentó el número de sus 
páginas, para pasar de folletos a libros; y la frecuencia de aparición, para llegar durante algún tiempo a cuatro números anuales, en estos momentos mantiene dos.

El pasado año 2006 completó el centenar de monografías y ya en el presente está en proceso de edición la 101 y en preparación la 102. Desde el número 80 está también en impresión digital, pero desde el 89 no se ha impreso en papel, lo que se trata de hacer nuevamente en estos momentos.

\section{RELACIÓN DE CUADERNOS PUBLI CADOS EN SUS 55 AÑOS DE EXI STENCIA:}

1. El Protomedicato de La Habana, por el Dr. Emeterio Santovenia (1952).

2. Centenario del nacimiento del Dr. Juan Guiteras Gener (1952).

3. El primer hospital de La Habana, por el Dr. Guillermo Lage (1952).

4. Oración Finlay, por el Dr. Enrique Saladrigas y Zayas (1952).

5. Epidemiología, por el Dr. José A. Martínez Fortún y Foyo (1952).

6. Historia de los hospitales y asilos de Puerto Príncipe o Camagüey (Período colonial), por el Sr. René Ibáñez Varona (1954).

7. La obra y la gloria de Finlay reconocidas por el XIV Congreso Internacional de Historia de la Medicina, por los Dres. Félix Hurtado, Horacio Abascal y César Rodríguez Expósito (1955).

8. Médicos en la vida de Martí, por el Sr. César Rodríguez Expósito (1955).

9. Reseña y sinonimia de la frambuesa y de la pelagra, por el Dr. Horacio Abascal (1955).

10. Centenario de la graduación del Dr. Carlos J. Finlay, en el J efferson Medical College, por el Sr. César Rodríguez Expósito (1956).

11. Permanencia de la doctrina de Finlay ante el XV Congreso Internacional de Historia de la Medicina, por el Dr. Horacio Abascal y el Sr. César Rodríguez Expósito (1957).

12. Vida estoica del Prof. Wilhelm Hoffman, por el Dr. Saturnino Picaza (1958).

13. Carlos J. Finlay, por el Sr. Sol Bloom (1959).

14. Médicos en la Sierra Maestra, por el Dr. Julio Martínez Páez (1959).

15. Dr. Carlos J. Finlay y el "Hall of Fame", por el Sr. César Rodríguez Expósito (1959).

16. La vida rural, por el Dr. Juan Santos Fernández (1960).

17. Finlay: polémica permanente, por el Sr. César Rodríguez Expósito (1961). 
18. Papeles del Dr. Juan Guiteras Gener, por el Sr. César Rodríguez Expósito (1962).

19. Cincuentenario de la muerte del Dr. Joaquín Albarrán, por el Sr. César Rodríguez Expósito (1962).

20. Finlay, por el Sr. César Rodríguez Expósito (1962).

21. Dr. Enrique Lluria Despau, por el Dr. Francisco Domenech (1963).

22. Dr. Ramón L. Miranda (Médico de Martí), por el Sr. César Rodríguez Expósito (1963).

23. Apuntes para la Historia de la Odontología en Cuba, por el Dr. José A. Martínez Fortún y Foyo (1963).

24. Apuntes para la Historia de los Hospitales de Cuba, por el Dr. Mario del Pino y de la Vega (1963).

25. La primera Secretaría de Sanidad del mundo se creó en Cuba, por el Sr. César Rodríguez Expósito (1964).

26. Bicentenario de Tomás Romay, por el Sr. César Rodríguez Expósito (1964).

27. Centenario del nacimiento del Dr. Francisco Domínguez Roldán, por la Sra. María Luisa Domínguez Roldán (1964).

28. Laura Martínez de Carvajal y del Camino (Primera graduada de Medicina en Cuba), por la Dra. María Julia de Lara (1964).

29. Papeles de Finlay, por el Sr. César Rodríguez Expósito (1965).

30. Centenario del nacimiento del Dr. Emilio Martínez y Martínez, por el Dr. Alfredo M. Petit (1965).

31. Dr. Francisco R. Argilagos, por el Dr. Rafael G. Argilagos (1965).

32. Dr. Claudio Delgado y su aportación al estudio de la fiebre amarilla, por el Dr. Ortelio Martínez Fortún y Foyo (1967).

33. Apuntes para la Historia de la Farmacia en Cuba, por los Dres. Manuel García Hernández y Susana Martínez Fortún (1967).

34. Dr. José A. Malberti, por el Dr. Emilio Teuma (1967).

35. Dr. Juan F. Dávalos: el sabio que sueña con las bacterias, por el Sr. César Rodríguez Expósito (1967).

36. Dr. Joaquín L. Dueñas, por el Dr. Angel Arturo Aballí (1967).

37. Centenario del nacimiento del Dr. Jorge Le Roy y Cassá, por el Dr. Raimundo de Castro y Bachiller (1968). 
38. Dr. Enrique Núñez y Palomino (En el cincuentenario de su muerte), por el Sr. César Rodríguez Expósito (1968).

39. Dr. José H. Pazos: gran entomólogo cubano, por el Dr. Manuel Arnau Macías (1968).

40. Índice de médicos, farmacéuticos, dentistas y estudiantes en la Guerra de los Diez Años, por el Sr. César Rodríguez Expósito (1968).

41. El Real Hospital Nuestra Señora del Pilar en el siglo XVIII (un hospital para los esclavos del Rey), por el Sr. Luis A. de Arce (1969).

42. Dr. Juan Manuel Sánchez de Bustmante y García del Barrio, por el Dr. Luis F. Le Roy y Gálvez (1969).

43. La donación de sangre en Cuba, por el Dr. Mario del Pino y de la Vega (1969).

44. Manuel Ramón Silva Zayas (médico, polígrafo, revolucionario y luchador antimperialista) (1866-1919), por el Sr. Jorge J uárez Sedeño (1969).

45. Dr. Enrique B. Barnet, por el Dr. José A. López del Valle (1969).

46. Dr. Oscar Amoedo y Valdés (Una figura de la odontología universal), por el Sr. César Rodríguez Expósito (1969).

47. La Medicina en La Habana (1550-1730), 1ra. Parte, por el Dr. J osé López Sánchez (1970).

48. La Medicina en La Habana (1731-1800), 2da. Parte, por el Dr. J osé López Sánchez (1970).

49. Las Ciencias Médicas en la Filatelia Cubana, por el Dr. Ernesto Bello Hernández (1970).

50. Dr. Nicolás Manzini y Carli, por el Dr. Miguel García Manzini (1970).

51. Dr. Manuel Sánchez Silveira (Médico Rural), por la Sra. Nidia Sarabia (1971).

52. Finlay por cuarta vez ante el Congreso Internacional de Historia de la Medicina, por el Sr. César Rodríguez Expósito (1971).

53. Dr. Francisco Etchegoyen y Montané (Padre de la Veterinaria Cubana), por el Dr. Luis F. Caballero León (1971).

54. Dr. Enrique López Veitía (Gran oftalmólogo y fundador de los congresos médicos de Cuba), por las Sras. Laura y Elisa López Carvajal (1971).

55. Dr. Félix Figueredo Díaz (Un hombre del 68 y de la Protesta de Baraguá), por el Sr. César Rodríguez Expósito (1973).

56. La Guerra de Cuba en 1878 (La Protesta de Baraguá), por el Dr. Félix Figueredo Díaz (1973). 
57. Regla: su aporte a la medicina cubana en el siglo XIX, por el Sr. Eduardo Gómez Luaces (1973).

58. Evocación de Paul Lafargue, por el Dr. Raúl Roa (1973).

59. Monografía histórica sobre San Diego de los Baños, por la Dirección Provincial de Salud Pública, Pinar del Río (1974).

60. Recuerdos de una larga vida, por el Dr. Mario E. Dihigo (1974).

61. Bio-bibliografía del doctor Jorge Le Roy y Cassá, por el Dr. Luis F. Le Roy y Gálvez (1976).

62. Dr. Idelfonso Pérez Vigueras, un cazador de parásitos, por el Dr. Luis F. Caballero León (1981).

63. Contemporáneos del Dr. Francisco Cabrera Saavedra, por varios autores (1981).

64. Médicos guerrilleros. Testimonios, por la Sra. Nidia Sarabia (1982).

65. La Doctrina Finlaísta: valoración científica e histórica a un siglo de su presentación, por el Dr. Gregorio Delgado García (1982).

66. Estudios sobre Historia Médica Cubana, por el Dr. Gregorio Delgado García (1983).

67. Dr. Nicolás J. Gutiérrez y Hernández. 1800-1890, por el Dr. Gregorio Delgado García (1984).

68. Revolución y tuberculosis, por el Dr. Gustavo Aldereguía Lima (1984).

69. Efemérides médicas cubanas, por la Dra. Elena López Serrano (1985).

70. Trabajos académicos y otros estudios, por el Sr. César Rodríguez Expósito (1985).

71. Autobiografía y otros estudios, por el Profesor Dr. Eugenio Torroella Mata (1986).

72. Temas y personalidades de la historia médica cubana, por el Dr. Gregorio Delgado García (1987).

73. Boletín de la Sociedad Cubana de Pediatría y de la Revista Cubana de Pediatría. Índices, por la Dra. Elena López Serrano (1988).

74. Medicina, docencia y política, por el Dr. Federico Sotolongo Guerra (1989).

75. Historia de la enseñanza médica superior en Cuba, por el Dr. Gregorio Delgado García (1990).

76. Estudios históricos y medicolegales, por el Dr. Francisco Lancís Sánchez (1991). 
77. Apuntes históricos de la salud pública en Pinar del Río, por la Sra. Milagros Fernández Vera y otros (1992).

78. El cólera morbo asiático en Cuba y otros ensayos, por el Dr. Gregorio Delgado García (1993).

79. Oficina del Historiador del Ministerio de Salud Pública y Cuadernos de Historia de la Salud Pública (40 Aniversario de su fundación), por los Dres. Gregorio Delgado García y Elena López Serrano (1994).

80. Primer Encuentro I beroamericano de Historiadores de la Salud Pública, por el Dr. Gregorio Delgado García (1995).

81. Conferencias de Historia de la Administración de Salud Pública en Cuba, por el Dr. Gregorio Delgado García (1996).

82. Máximo Zertucha, médico del Lugarteniente General Antonio Maceo, por Gregorio Delgado Fernández y Rafael Soto Paz (1997).

83. La Salud Pública y la Juventud en el pensamiento del Che Guevara, por los Drs. Gregorio Delgado García y Elena López Serrano (1997).

84. En los dominios de Esculapio, por el Dr. Gregorio Delgado García (1998).

85. La Sanidad Militar del Ejército Libertador de Cuba, por los generales Eugenio Sánchez Agramonte y Eugenio Molinet Amorós (Infomed) (1999).

86. Los cubanos y los Premios Nobel, por el Dr. Gregorio Delgado García y Lic. J osé A. López Espinosa (1999).

87. Caduceos y Juramentos Médicos, por el Dr. Raimundo de Castro y Bachiller (2000).

88. Solidaridad Internacional de la Medicina Cubana. Testimonios, por el Dr. Washington Rosell Puig y otros (2000).

89. Estudios sobre el doctor Eugenio Espejo, por el profesor Manuel I. MonterosValdivieso (Infomed) (2001).

90. Oficiando ante Higea, por el Dr. Gregorio Delgado García (Infomed) (2001).

91. Efemérides y Noticias Médicas Cubanas (1959-1999), por la Dra. Elena López Serrano (Infomed) (2002).

92. Dr. Arístides Agramonte Simoni (1868-1931), por el Dr. Jorge Le Roy Cassá (Infomed) (2002).

93. Dr. Ángel A. Aballí Arellano (1880-1952), Maestro de la Pediatría Cubana, por los Drs. Daniel Alonso Menéndez y Gregorio Delgado García. (Infomed) (2003).

94. Testimonios de médicos revolucionarios cubanos, por los Drs. Faustino Pérez Hernández, Héctor Vera Acosta y otros (Infomed) (2003). 
95. Evocaciones del pasado médico cubano, por el Dr. Gregorio Delgado García (Infomed) (2004).

96. Historia de la Medicina en Cuba. Siglos XVI a primera mitad del XVIII, por el Dr. José A Martínez- Fortún Foyo (Infomed) (2004).

97. Historia de la Medicina en Cuba (1751-1839), por el Dr. José A Martínez- Fortún Foyo (Infomed) (2005).

98. Historia de la Medicina en Cuba (1840-1958), por el Dr. José A Martínez- Fortún Foyo (Infomed) (2005).

99. Relieve de Historia Médica Cubana, por el Dr. Gregorio Delgado García (Infomed) (2006).

100. La Nacionalización de los Servicios de Sanidad y otros estudios, por el Dr. José A. López del Valle y Valdés (Infomed) (2006).

101. Los Hogares Maternos de Cuba, por los Dres. José A. Gutiérrez Muñiz y Gregorio Delgado García (Infomed) (2007).

102. EL Empacho en América Latina y Cuba y otros estudios, por el Dr. Roberto Campos Navarro (Infomed) (2007).

* El Dr. Gregorio Delgado García es el Director de Cuardenos de Historia de la Salud Pública desde 1972. 